\title{
Physical exercise as a tool to help the immune system against COVID-19: an integrative review of the current literature
}

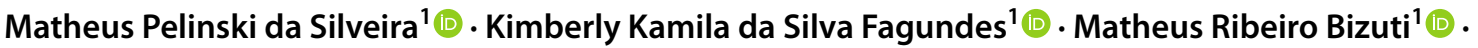 \\ Édina Starck ${ }^{1}\left[\right.$ - Renata Calciolari Rossi ${ }^{2} \odot$. Débora Tavares de Resende e Silva ${ }^{1}(1)$
}

Received: 9 June 2020 / Accepted: 24 July 2020 / Published online: 29 July 2020

(c) Springer Nature Switzerland AG 2020

\begin{abstract}
Acute viral respiratory infections are the main infectious disease in the world. In 2020, a new disease caused by severe acute respiratory syndrome coronavirus 2 (SARS-CoV-2), coronavirus disease 2019 (COVID-19), became a global pandemic. The immune response to the virus depends on factors such as genetics, age and physical state, and its main input receptor is the angiotensin-converting enzyme 2. The practice of physical exercises acts as a modulator of the immune system. During and after physical exercise, pro- and anti-inflammatory cytokines are released, lymphocyte circulation increases, as well as cell recruitment. Such practice has an effect on the lower incidence, intensity of symptoms and mortality in viral infections observed in people who practice physical activity regularly, and its correct execution must be considered to avoid damage. The initial response is given mainly by type I interferons (IFN-I), which drive the action macrophages and lymphocytes, followed by lymphocyte action. A suppression of the IFN-I response has been noted in COVID-19. Severe conditions have been associated with storms of pro-inflammatory cytokines and lymphopenia, as well as circulatory changes and virus dispersion to other organs. The practice of physical activities strengthens the immune system, suggesting a benefit in the response to viral communicable diseases. Thus, regular practice of adequate intensity is suggested as an auxiliary tool in strengthening and preparing the immune system for COVID-19. Further studies are needed to associate physical exercise with SARS-CoV-2 infection.
\end{abstract}

Keywords COVID-19 $\cdot$ Exercise $\cdot$ Immune system $\cdot$ Coronavirus

Débora Tavares de Resende e Silva

debora.silva@uffs.edu.br

Matheus Pelinski da Silveira

matheuspelinski@gmail.com

Kimberly Kamila da Silva Fagundes

kymberly-k@hotmail.com

Matheus Ribeiro Bizuti

matheus_ribeiro.bizuti@hotmail.com

Édina Starck

edina.starck@hotmail.com

Renata Calciolari Rossi

renatacalciolari@terra.com.br

1 Federal University of Fronteira Sul, Campus Chapecó, SC 484 - KM 02, 89802-000 Chapecó, SC, Brazil

2 University of Oeste Paulista (UNOESTE), Presidente Prudente, Brazil

\section{Introduction}

Acute respiratory infections (ARIs) are caused by respiratory viruses and bacteria, being the most infectious disease in humans $[1,2]$. These can be caused by more than 200 different viruses, with rhinovirus being the most common etiological agent [3-5]. In December 2019, a new coronavirus outbreak was reported in China, being called the Severe Acute Respiratory Syndrome Coronavirus 2 (SARS-CoV-2), spreading rapidly and infecting more than 14 million people, being declared a Health Emergency International Public Service on January 30, 2020 [6, 7].

The main mode of transmission is contact with droplets containing viral particles eliminated through the cough or sneeze of an infected person, and the incubation period usually varies from 2 to 14 days. Approximately $80 \%$ of the cases are asymptomatic or with mild symptoms, and the others can be severe or critical and can lead to death [8]. The development of Coronavirus Disease 2019 (COVID-19) is 
dependent on the interaction between SARS-CoV-2 and the host's immune system, the immune response being influenced by genetics (HLA genes), age, sex, nutritional status and status physical [9].

The immune response includes two stages, innate immunity and adaptive immunity. The first one comprises physical and chemical barriers and the action of cells such as macrophages, dendritic cells (DCs), natural killer cells (NKs), neutrophils and molecules such as cytokines, interleukins (ILs), nitric oxide (NO) and superoxide anion (O2-). The second one has as mechanism of action the T lymphocytes (TCD4 + and TCD8 +) and B lymphocytes and their products, such as antibodies and cytokines. Furthermore, the adaptive immune response can be subdivided into cellular immunity (mediate by cells as macrophages and lymphocytes) and humoral immunity (mediates by cells as macrophages and lymphocytes) and humoral immunity (mediated by antibodies) $[10,11]$. The regular practice of physical exercises promotes improvements in quality of life and can act in the immune response, reducing the risk of developing systemic inflammatory processes and stimulating cellular immunity [12].

Therefore, the present article aims to perform an integrative review of the literature relating the role of physical exercise on the immune system in the fight against COVID-19. For this purpose, the bibliographic study included knowledge about respiratory infections, influences of physical exercise on the immune system and proposed the comprehension of the most recent information about the immunopathogenesis of SARS-CoV-2 infection, also comprising its relationship with the host's physical and health conditions.

\section{Influence of physical exercise on the immunological response}

Physical activity is considered one of the main components of healthy living. In addition to the functions related to the prevention of excess body weight, systemic inflammation and chronic non-communicable diseases, a potential benefit of physical exercise in reducing communicable diseases, including viral pathologies, is suggested [13].

The practice of physical exercise, both in its acute form and in its chronic form, significantly alters the immune system $[14,15]$. Studies indicate that the modulation of the immune response related to exercise depends on factors such as regularity, intensity, duration and type of effort applied $[13,16]$.

Moderate-intensity physical exercises stimulate cellular immunity, while prolonged or high-intensity practices without appropriate rest can trigger decreased cellular immunity, increasing the propensity for infectious diseases [14, 15]. According to the International Society for Exercise and
Immunology (ISEI), the immunological decrease occurs after the practice of prolonged physical exercise, that is, after 90 min of moderate- to high-intensity physical activity [17]. Cellular changes due to physical activity are illustrated in Fig. 1.

\section{Effects of physical exercise on immune system components}

\section{Cytokines}

Cytokines are classified as anti-inflammatory and proinflammatory according to their functions. Among the antiinflammatory cytokines, we highlight IL-10 and transforming growth factor-beta (TGF- $\beta$ ), responsible for inhibiting the production of pro-inflammatory cytokines [18]. Among the pro-inflammatory cytokines, we highlight IL-1, IL-2, IL-12, IL-18, interferon-gamma (IFN- $\gamma$ ) and tumor necrosis factor-alpha (TNF- $\alpha$ ) [19]. Cytokine production can be modified due to hormonal or oxidative stress and physical exercise. The muscle contraction has the effect of increasing the release of antinflammatory and pro-inflammatory cytokines at levels that vary according to the volume of contractile mass involved, duration and intensity of exercise [20].

\section{Neutrophils}

During the practice of physical exercise, the activation of the muscle fiber is responsible for increasing the release of calcium $(\mathrm{Ca} 2+)$ and, therefore, promoting the synthesis of proinflammatory cytokines, namely TNF- $\alpha$ and IL- $1 \beta$, which act in the regulation of selectins, which, in turn, attract neutrophils to the site [21]. The neutrophilia induced by physical activity is due to the release of neutrophils from the bone marrow due to the influence of cortisol [22].

After aerobic physical exercise (approximately $24 \mathrm{~h}$ ), there is a significant reduction in neutrophil chemotaxis, however, without compromising bactericidal activity. The reduction in neutrophil chemotaxis is reversed within $48 \mathrm{~h}$ after physical activity, during which the opportunistic activity of infectious microorganisms can occur [23].

\section{Leukocytes}

Physical activity is also responsible for increasing the concentration of circulating leukocytes [24]. This is due to shearing of immune cells in blood vessels, especially secondary lymphoid tissues such as liver, spleen and lung [25]. The leukocyte concentration remains high with a peak of 30-120 min after constant physical activity, which may persist for up to $24 \mathrm{~h}$ after [24]. 


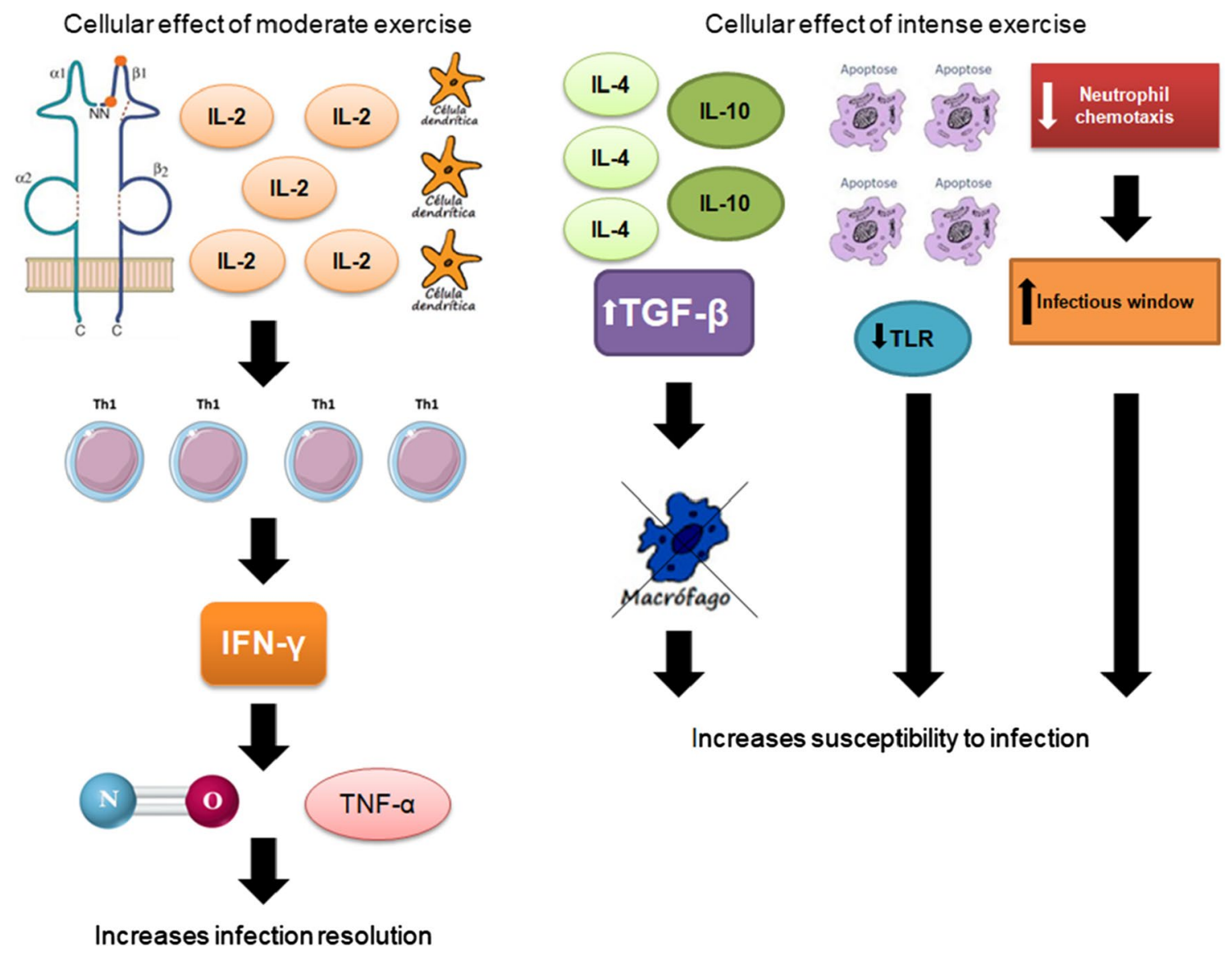

Fig. 1 Effect of physical activity on the immune system. Source: The Authors (2020)

\section{Antigen-presenting cells (APCs)}

The practice of aerobic physical exercise in an exacerbated manner is responsible for decreasing the expression of Tolllike receptors (TLRs) in macrophages, considerably reducing the presentation of antigens to $\mathrm{T}$ lymphocytes, thus causing the suppression of the inflammatory $\mathrm{T}$ helper type 1 (Th1) response. Thus, the failure to develop an inflammatory activity precludes possible tissue damage resulting from inflammatory mediators and, consequently, the risk of chronic inflammatory processes. However, the susceptibility to infections due to intracellular microorganisms increases [26].

\section{Natural killer cells (NKs)}

During physical activity, blood flow increases in order to supply the metabolic demands of the human body. The recruitment of NK cells occurs through cellular stress promoted by exercise and a consequent decrease in adhesion molecules induced by catecholamines [27]. However, physical activity lasting more than three hours causes the concentration of NK cells to return to the pre-exercise state or even lower than this. This is because the NK cells migrate to the muscle injury site [28].

\section{Lymphocytes}

During moderate physical exercise, the concentration of lymphocytes increases in the vascular bed and, after strenuous exercise, decreases to levels below the pre-exercise period $[29,30]$. The CD4 +:CD8 + ratio decreases as TCD8 + cells increase [14].

TCD4 + cells decrease due to the increase in NK cells $[14,31]$. After physical activity, the lymphocyte concentration decreases due to the apoptosis mechanism [32]. Thus, the increase in lymphocyte concentrations favors the Th1mediated immune response, preventing infections by intracellular microorganisms [24] (Fig. 2).

\section{SARS-COV-2 virology and the role of the immune system in COVID-19 infection}

SARS-CoV-2 is constituted by single-strand positivesense RNA and belongs to the genus Betacoronavirus, lineage $B$ and subgenus Sarbecovirus. Viral genome 


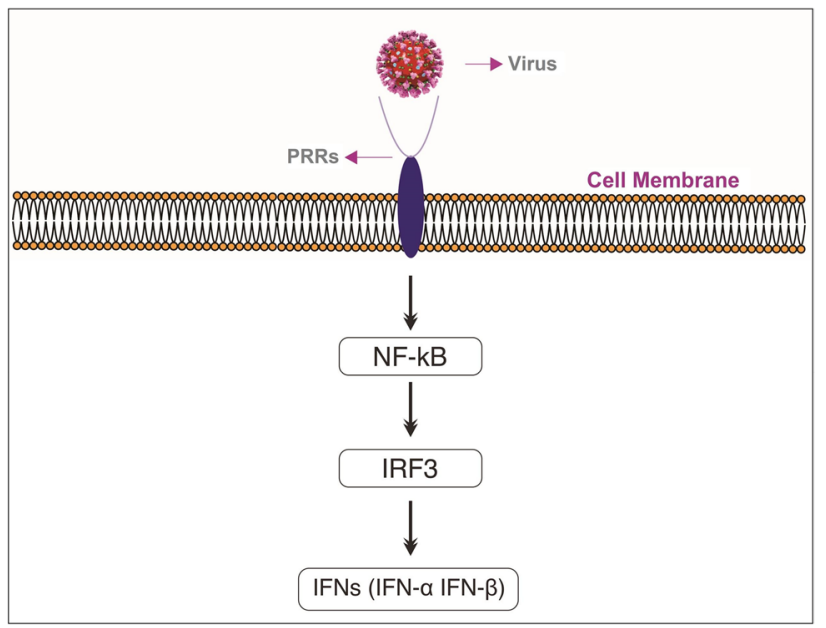

Fig. 2 Innate immune response to the presence of viruses. Source: The Authors (2020)

studies have identified similarity of SARS-CoV-2 with bat coronaviruses, as well as coronaviruses responsible for two previous pandemics: the Severe Acute Respiratory Syndrome-Related Coronavirus (SARS-CoV) and the Middle Eastern Respiratory Syndrome Coronavirus (MERS-CoV) [33, 34].

Similar to SARS-CoV, the novel coronavirus uses a structural glycoprotein to infect cells: the envelope Spike protein (S), using the Angiotensin-Converting Enzyme-2 (ACE2) as entry receptor [33,34]. ACE2 consists of a cell membrane protein abundantly expressed in the organism, which is present in cardiac, pulmonary, renal, intestinal and vascular cells, and the binding of SARS-CoV-2 with this enzyme has a strong affinity, which may explain the high transmissibility of the virus [8, 9, 34].

Due to mainly respiratory symptoms, it is believed that the target cells of SARS-CoV-2 are in lower airways [35], as is the case of type 2 pneumocytes or alveolar cells, the main site of expression of ACE2 receptors [8]. The result of the interaction between the $\mathrm{S}$ receptor binding domain (RBD) and ACE2 is the fusion of the viral and host membranes, which proceeds for viral replication and dissemination and may reach the other cells with ACE2 expression in the organism [8].

With the purpose of containing the infection, the innate and adaptive immune system is activated by mechanisms that are not yet completely elucidated. In spite of this, it is known that effective immunological actions are essential to control viral replication and dissemination, cellular inflammation and tissue injury, and many studies have reported that the immune response of the host influences the severity of COVID-19 [9, 36-38].

\section{Immune response to coronavirus infections}

To initiate the antiviral response, cells of the innate immune system need to recognize the infection, a process performed through pattern-recognition receptors (PRRs) such as TLR, NOD-like receptor (NLR), C-type lectinlike receptor (CLR), RIG-I-like receptor (RLR) and freemolecule receptors in the cytoplasm, which detect pathogen-associated molecular patterns (PAMPs). Once viral nucleic acids are recognized as PAMPs, PRRs activate molecular pathways of inflammatory response, stimulating chemotaxis, maturation of immune cells, phagocytosis and expression of inflammatory factors [39].

Viral recognition by TLR3, TLR7 and RIG-I receptors leads to activation of the nuclear factor- $\mathrm{KB}(\mathrm{NF}-\mathrm{\kappa B})$ and IRF3 signaling cascade, with nuclear transcription and expression of type I interferons (IFNs-I) and pro-inflammatory cytokines, creating the first line of defense against viral infections. The IFNs-I (IFN- $\alpha$ and IFN- $\beta$ ) are the most important antiviral cytokines, and they act as immunomodulators influencing the activities of macrophages and lymphocytes, performing actions such as protection of non-infected cells, containment of viral replication and effective activation of the adaptive immune system $[8,9$, 39].

A suppression or delay of IFNs-I response-due to viral evasion mechanisms - results in impairment of early infection control, hyperinflammatory infiltrate of neutrophils, macrophages and monocytes into the lungs, production of cytokines by these cells and lung tissue damage. This process, described in SARS-CoV and MERS-CoV, has been suggested as a possible strategy to trigger or collaborate to the pathology of COVID-19 [8,9].

Macrophages and DCs act as APCs for lymphocytes via MHC and produce a microenvironment of signaling cytokines, activating the adaptive immune system. $\mathrm{T}$ lymphocytes perform important functions against viral microorganisms, since TCD8 + can cause direct cytotoxicity against infected cells and TCD4 + stimulate B lymphocytes to produce neutralizing antibodies. In turn, $\mathrm{T}$ helper lymphocytes (Th) - predominantly Th1—contribute to the organization of the adaptive response and release cytokines that are able to recruit monocytes and neutrophils and to promote other cascades of pro-inflammatory molecules, amplifying the immune response $[8$, 39] (Fig. 3).

The complement system can also be activated and has an important role in coronavirus infections, because it helps the innate immune system to identify antigens. However, its activation can contribute to the disease due to its potent capacity to stimulate neutrophils and to recruit inflammatory cells, which can trigger tissue damage [39]. 


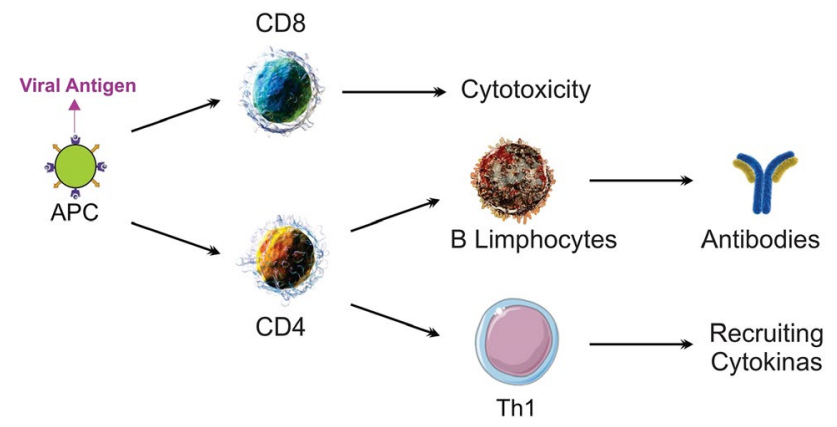

Fig. 3 Adaptive immune response to the presence of viruses. Source: The Authors (2020)

\section{Unbalanced immunological responses at COVID-19}

Studies suggest that in mild cases, pulmonary tissue macrophages are capable of containing SARS-CoV-2 and innate and adaptive immune responses are efficiently activated against viral replication. However, severe cases of COVID19 are associated with an imbalance in antiviral immunity, characterized by two main situations: a pro-inflammatory cytokine storm and a lymphopenia state [37,38].

The degree of lymphopenia and cytokine storm was related to the severity of COVID-19 [7], and similar situations had already been reported in other respiratory viral infections, including influenza, SARS-CoV and MERS-CoV. Activation of the complement system and abnormalities in coagulation were also observed in severe patients with markers such as C-reactive protein (CRP), dimero-D and fibrin degradation products, which are usually elevated in advanced stages of the disease [37, 38].

The mechanism of cytokine storm and lymphopenia associated with circulatory alterations and viral dissemination to several organs was proposed as being responsible for viral sepsis. Among the complications of this condition are acute respiratory distress syndrome (ARDS), septic shock, multiple organ failure (MOF) and death $[8,38]$.

\section{Cytokine storm}

In patients with severe COVID-19, increased levels of cytokines were observed, including IL-1 $\beta$, IL-2, IL-6, IL-8, IL-10, IL-17, interferon-gamma (IFN- $\gamma$ ), tumor necrosis factor alpha (TNF- $\alpha$ ), granulocyte-colony stimulating factor (G-CSF), gamma-induced protein 10 (IP10), monocyte chemoattractant protein 1 (MCP1), macrophage inflammatory protein 1 alpha (MIP1- $\alpha$ ) and other molecules, characterizing the cytokine storm $[37,38]$. Additionally, elevations of IL- $1 \beta$, IFN- $\gamma$, IP10 and MCP1 in infections by the novel coronavirus were associated with the Th1 response; however, an increase in interleukins of the T helper type 2 (Th2) profile, such as IL-4, IL-5, IL10, which suppress the inflammation, was also associated with a greater severity of COVID-19, which may demonstrate an imbalance in immune regulation and an attempt to minimize tissue inflammatory damage $[35,40]$.

The cytokine storm generates an immunological system attack against the organism, which can cause substantial lesions in organs such as the lung, heart, brain, kidneys, spleen, liver and lymph nodes. The increase of neutrophils, macrophages and monocytes in association with a dysfunction of the IFN-I response has been reported as the main cause of lethality in SARS-CoV and MERS-CoV pneumonia, and a similar conclusion has been suggested for SARSCoV-2 [9, 37, 38].

In the lungs, alveolar macrophages and epithelial cells are the most responsible for the production of cytokines and chemokines. During infection by the novel coronavirus, excessive secretion of these molecules by the immune cells mediates a massive pulmonary infiltrate of neutrophils, monocytes and macrophages, which results in alveolar damage due to wall thickening and formation of hyaline membranes. In addition, the accumulation of neutrophils in the lungs increases the production of reactive oxygen species (ROS) and pro-inflammatory molecules, predisposing to injury [41]. Therefore, high levels of pro-inflammatory cytokines are associated with respiratory insufficiency, ARDS and may lead to shock, MOF and death in the COVID-19 [37, 38].

In comparison, pulmonary disease caused by SARS-CoV also presents with formation of hyaline membranes, desquamation of alveolar space pneumocytes and interstitial infiltration with lymphocytes and mononuclear cells. In the serum of patients who develop SARS, there are high levels of cytokines and pro-inflammatory chemokines [42].

Additionally, it has been observed that during viral infections, pro-inflammatory cytokines can stimulate an increase in the levels of ACE2 protein, the receptor for SARS-CoV-2. A larger quantity of this protein may accelerate the entry of the novel coronavirus into the host cells and contribute to its dissemination in the organism, negatively influencing the antiviral response [34].

\section{Changes in cellular counts}

In critically ill patients, a state of immune suppression is also described, with a significant and sustained decrease in the absolute number of TCD4 + and TCD8 + lymphocytes, possible reduction of $\mathrm{B}$ lymphocytes, NK cells, monocytes, eosinophils and basophils [37,38]. The study conducted by Liu et al. [7] did not observe significant changes in the total counts of B lymphocytes, NK cells and monocytes; however, there was a considerable reduction in the lymphocyte count in peripheral blood of patients with severe COVID-19 at the 
beginning of the disease, especially cytotoxic TCD8 + cells [7].

Activation markers for TCD4 + and TCD8 + lymphocytes showed excessive stimulation and exhaustion markers for TCD8 + lymphocytes were elevated in the disease, suggesting lymphocyte dysfunction [9, 37]. Additionally, an increase in the number of neutrophils and a greater neutrophil-to-CD8 + T cell ratio (N8R) was associated with severe COVID-19 and proposed as the most significant predictor of poor prognosis [7, 37].

Cell death induced by interaction between Fas and Fas ligand by activation of the TNF-related apoptosis inducing ligand axis and by direct infection of $\mathrm{T}$ lymphocytes by SARS-CoV-2 may be responsible for the origin of lymphopenia in COVID-19 [38]. The cytokine storm can also influence the lymphopenia, since in the study of Liu et al. [7], the peaks in cytokine levels IL-2, IL-4, IL-10, TNF- $\alpha$ and IFN- $\gamma$ coincided with the lowest T lymphocyte counts, about 4-6 days after the onset of severe COVID-19; therefore, the restoration of $\mathrm{T}$ cell numbers was associated with reductions in circulating cytokines [7].

The fact that $\mathrm{T}$ cells are important regulators of the activation of the immune system during a viral infection may explain how lymphopenia is related to the worsening of inflammatory responses [7]. In consequence of lymphopenia and lymphocyte dysfunction, the adaptive immune response is ineffective and the infection is not adequately controlled, further increasing the stimulation of cytokines and cellular infiltrations [38].

\section{Coagulation disorders}

It was observed that patients with SARS-CoV-2 infection present increased risk of venous thromboembolism (VTE) and disseminated intravascular coagulation (DIC). Coagulopathy associated with COVID-19, as it may be named, is characterized by hypercoagulability and thrombosis and is associated with worse prognosis in infection. Among the altered coagulation parameters in patients with severe COVID-19 are exacerbated coagulation activation, coagulation factor consumption, prolongation of prothrombin time (PT) and activated partial thromboplastin time (aPPT), moderate to severe thrombocytopenia, increased D-dimer and reduction of fibrinogen [43].

Through a retrospective analysis of 183 patients with coronavirus pneumonia, high levels of the D-dimer and fibrin degradation product have been identified, in addition to prolonged PT, as well as aPPT in patients who had deceased. Tang et al. [44] considered the D-dimer as an important coagulopathy marker in cases of SARS-COV-2 infection. The same findings were found in the studies by
Han et al. [45] who see the use of hemostasis tests as tools to be used in early diagnosis and in monitoring disease progression [46].

Blood coagulation is the fastest mechanism in the confinement and inactivation of infections, being the first and the last defense line of the innate immune system to take place in tissues and blood circulation. The sore promotes the activation of the endothelial cells and the dysfunction of the endothelium, thus generating a pro-thrombotic state [47]. In cases hyperactivation of the immune system, the coagulation may become intravascular and disseminated, therefore causing multiple organ failure. After all, the amount of vascular endothelial lesions of organs and tissues is, due to their activation intent, inversely proportional to the amount of existing coagulation factors [48].

Thereupon, the first clinical studies carried out in patients with pneumonia caused by the Coronavirus confirmed the occurrence of organ dysfunction and coagulopathy as possible causes of the negative outcomes of the disease [44]. The activation of the immune system, in response to the infection, leads the production of cytokines and tissue factor expression. The cytokines, in large amounts, harm the gas exchange and lead not only to inflammation but fibrinolysis, thus increasing D-dimer concentration [49].

The tissue factor is related to an increase in thrombin generation and fibrin deposition, leading to hypercoagulability and CIVD and, thus, a worse prognosis [46, 49]. As a contributor to the coagulation process, there is the presence of polymorphonuclear leukocytes (PMN) that are activated during the inflammatory process, releasing extracellular neutrophil traps (NETs) which contain proteases that generate the inactivation of endogenous anticoagulants and the propagation of a procoagulant state. The interaction of activated platelets with PMN can form vaso-occlusive thrombotic complexes [47].

Some authors also elucidate the virus's relationship with the ECA2 functional receptor, present in the arterial and venous endothelial cells of most human organs and part of both axes of the renin-angiotensin system, the vasoconstrictor ECA/Ang/AT1R and the vasodilator ECA2/Ang-(1-7)/MAS [50, 51]. Dalan et al. [52] cite that both aging and metabolic disorders positively regulate the ECA/Ang/AT1R axis, leading to inflammatory, oxidative, vasoconstrictor and fibrotic effects. Therefore, the ECA2/ Ang-(1-7)/MAS axis is negatively regulated, resulting in a decrease in the anti-inflammatory and anti-fibrotic effects [52]. The connection of SARS-COV-2 to the ECA2 receiver affects the balance between ECA/Ang/AT1R and ECA2/Ang-(1-7)/MAS, making the effects of the ECA/ Ang/AT1R axis even more prominent [53]. 


\section{Host condition and COVID-19}

\section{Aging}

The different immune responses of the host to the SARSCoV-2 infection may explain the reason why men and women, young and old, infected by the virus can suffer a different severity of the disease [54]. Therefore, a considerably higher mortality rate was observed in patients with advanced chronological age [55].

Immune aging is related to an increase in individuals' susceptibility to infections, due to the decline in immune function, which can occur at any stage of the immune response. Such changes can be seen, especially when associated with emotional stress [56]. Immune senescence is associated with the suppression of the activation and presentation of antigens by macrophages, which consequently prevent the migration of dendritic cells and the activation made by Toll receptors with less effect [56, 57]. Ewers et al. [58] mention the decline and proliferation of $\mathrm{T}$ cells, in addition to the increased production of pro-inflammatory cytokines IL-1, IL- 6 and TNF- $\alpha$ in the elderly.

Another point is the imbalance between Th1 and Th2 cytokines, generating an increase in the susceptibility of these individuals as infections by viruses and extracellular bacteria [58]. In view of this, aging is associated with a constitutive pro-inflammatory environment due to persistent and low-grade immune activation, which can lead to increased tissue damage caused by infections [57].

Also, there should be taken into account the positive regulation of the ACE-Ang-II-AT axis that leads to proinflammatory and pro-fibrotic effects. Although not very detailed, it is still suggested the occurrence of a greater number of ECA2 receptors associated with aging, which would increase this imbalance. After all, it is through this receptor that SARS-COV-2 infects humans and thus contributes to the development of COVID-19 in older people [52].

\section{Obesity, type 2 diabetes mellitus (T2DM) and metabolic syndrome (MS)}

The precarious metabolic health is considered the main risk factor for the development of severe forms of COVID-19. This may occur in T2DM, obesity and MS, possibly due to immune dysfunction in synergism with pathophysiological complications of these comorbidities [59].

Increased ACE2 expression is a protective adaptive mechanism in T2DM; however, it may facilitate the viral entry and spread of SARS-CoV-2 in the body [59]. Adipose tissue also exhibits high expression of ACE2, so the population with obesity may present greater vulnerability to COVID19 [43].
It was observed that the expression of ACE2 in the adipose tissue of obese patients allows viral entry in adipocytes and makes this tissue a reservoir for the viral dissemination of SARS-CoV-2, since it is viscerally distributed $[43,51]$. In addition, obesity is an important factor for the development of T2DM-especially when associated with low levels of physical activity and poor physical conditioning - and as mentioned, both diseases are related to higher expression of ACE2, increasing the risk of advanced infection by SARSCoV-2 [43].

Metabolic disorders lead to immune activation of tissues such as the adipose, increasing the concentration of lowgrade chronic inflammation plasma markers, called metabolic inflammation or meta-inflammation [61]. In this sense, the release of pro-inflammatory adipokines such as leptin, TNF- $\alpha$, IL- 6 and IL- $1 b$ is observed, with a reduction in antiinflammatory action through the suppression of adiponectins $[62,63]$. The presented relationship is directly proportional to the presence of adipose tissue, the same that can be regulated through the practice of physical activity [64].

The pro-inflammatory state found in metabolic syndrome and T2DM may increase the probability of an unbalanced inflammatory response in COVID-19, like the cytokine storm described in patients with severe disease [59]. Similarly, as obesity is a state of low-grade chronic inflammation, it shows a potential for immune amplification of pathogens, as the regulatory elements of the immune response are absent or dysfunctional and this may contribute to the cytokine storm, which is already in greater concentration in obese individuals and which sustains and activates multiple cytokine pathways for a long time after the viral insult $[65$, 66].

Damage to blood vessels caused by chronic diseases, such as T2DM, associated with hypercoagulability present in COVID-19 may intensify the risk of infection complications [59]. Obesity can also aggravate endothelial dysfunction present in COVID-19 due to inflammation triggered by perivascular and vascular adipose tissue, combined with changes in the synthesis of endogenous vasoactive agents, leading to platelet hyperactivation, leukocyte adhesion and other modifications related to endothelial inflammation, prothrombosis and proatherogenesis [43].

Hypercoagulability is directly proportional to the severity of overweight in obese patients. Among the pathophysiological mechanisms are the action of adipocytokinins, with leptin and adiponectin, overactivity of coagulation factors, reduction of fibrinolytic function and, once again, increased inflammation (TNF and IL-6). Other contributors include elevated oxidative stress, lipid and glucose tolerance disorders, MS and venous stasis. Thus, it is considered a synergic effect of obesity and MS on the state of hypercoagulability in COVID-19, aggravating the risk of VTE and DIC even more [43]. 
There is also a greater amount of macrophages in the adipose tissue of obese individuals due to areas of microhypoxia, which lead to the nuclear factor- $\kappa \mathrm{B}(\mathrm{NF}-\kappa \mathrm{B})$ pathway activation, thus increasing the expression of genes involved in inflammation [61]. This condition is the result of the attraction of monocytes in the circulation made by chemokines; when they infiltrate the adipose tissue, they transform into macrophages, which, in turn, release TNF- $\alpha$ and IL-6 which induce the tissue's resistance to insulin [63]. Insulin resistance is also related to the host's immune response, as it can inhibit the resolution of $\mathrm{T}$ cell-mediated inflammation [61].

The adipose tissue is not the only one affected by the deposition of fats, because of that, bone marrow and thymus also present significant changes to the immune system of obese individuals and those with MS [67]. Thus, there is a marked deregulation of immune responses, which leads to a lower presence of circulating $\mathrm{T}$ cells, reducing the response to pathogens $[60,61,65]$.

\section{Physical exercise and COVID-19}

Despite the lack of accurate data on how physical activity improves the immune response against the new coronavirus, there is evidence of lower rates of ARI incidence, duration and intensity of symptoms and risk of mortality from infectious respiratory diseases in individuals who exercise at high levels appropriate. Furthermore, different studies suggest that regular physical exercise is directly related to decreased mortality from pneumonia and influenza, improvements in cardiorespiratory function, vaccine response, metabolism of glucose, lipids and insulin $[13,16]$.

Increased immune surveillance against infections has been proposed as a mechanism responsible for improving the immune response related to physical exercise. Moderateintensity physical activity is responsible for providing an increase in the anti-pathogenic activity of macrophages, at the same time as elevations in the circulation of immune cells, immunoglobulins and anti-inflammatory cytokines occur, thereby reducing the burden of pathogen on organs such as the lung and the risk of lung damage due to the influx of inflammatory cells [12].

During regular physical exercise practices, inflammatory responses and stress hormones are decreased; in contrast, lymphocytes, NK cells, immature B cells and monocytes are at high levels. Thus, there is an improvement in immunovigilance, as well as a reduction in the systemic inflammatory process, factors that corroborate that regular physical activity helps to improve the immune system, while helping to prevent respiratory diseases and thus protect against infections such as COVID-19 [68].
Dynamic exercises that generate cardiorespiratory overload promote the mobilization and redistribution of effector lymphocytes, mediated by catecholamines. This action primarily stimulates subtypes of lymphocytes capable of migrating from reservoirs_-such as blood vessels, spleen and bone marrow - to lymphoid tissues and organs-such as the upper respiratory tract, lungs and intestines, aiming at recognizing and fighting pathogens and, thus, increasing immune surveillance and improving the antiviral response [16].

Similarly, regular exercise practices at moderate levels favor the function of the human body's immune surveillance against pathogens, as they stimulate an exchange of white blood cells between the circulatory system and tissues, a fact that reduces morbidity and mortality from acute respiratory disease and infections viral. They are also capable of promoting protection against infections triggered by intracellular microorganisms, as viral agents, given that the predominant immune response is mediated by Th1 cells [68].

Regular exercise of moderate intensity has already been associated with a reduction in respiratory infections compared to sedentariness. However, exhaustive physical practices before or during an infectious condition, such as influenza or COVID-19, can trigger severe illness due to changes in the immune system $[40,68]$. This occurs due to the production of Th2 anti-inflammatory cytokines in order to reduce muscle tissue damage, but in strenuous activities this effect can reach immunosuppression levels, thus providing the opportunity for infections $[12,40]$. Therefore, attention should be paid to the importance of developing physical training at appropriate levels of execution.

To the detriment of the world demographic change and the habits arising from the technological revolution, the population is aging more, becoming more obese and, consequently, less active when it comes to physical exercise. In this way, the immune system undergoes negative changes; that is, there is a functional impairment of innate immunity and adaptive immunity called immunosenescence, which results in greater susceptibility to infectious diseases and systemic inflammatory processes, decreased response to antibodies and, therefore, compromised immunological surveillance [68].

Therefore, for the elderly population, physical activity is even more essential, as these individuals generally have greater comorbidities and, in relation to the new coronavirus, are more vulnerable to contracting the disease [69]. Damiot et al. [70] suggested that individuals who have remained active throughout their lives have less pronounced immunosenescence characteristics, which may be a possible protective factor against the development of complications caused by COVID-19.

In this sense, beneficial effects of regular physical exercise have been reported in the elderly population, including 
reduction in oxidative stress, improvement in immune competence and reduction in cellular changes related to immunosenescence $[13,16]$. Elderly individuals who maintain continued physical activity have levels of TCD4 + and TCD8 + lymphocytes similar to younger individuals, in addition to not having harmful defects in the recruitment of lymphocytes during the infectious process [58].

According to the study by Ferrer et al. [71] with 116 elderly volunteers, through physical activity there is a decrease in the current levels of IL-6, as well as an increase in the expression of IL-10 in active individuals [71]. A low presence of circulating pro-inflammatory cytokines is observed in contrast to the increase in anti-inflammatory cytokines. Thus, there are positive changes in the immune system of these individuals, including enhancements in host response and vaccine immunoprotection [70].

Similarly, while prolonged maintenance or worsening of obesity and MS perpetuates deregulation of immune responses, promoting greater risks of the individual developing diseases and increasing their vulnerability to infection by the novel coronavirus [43, 59, 61]. An association between physical activity and reduction of inflammatory markers in obese and overweight patients is suggested [13].

Luzi and Radaelli [72] add the lack of physical activity as an important factor among obese patients, as it impairs the immune response against microbial agents, from the activation of macrophages to the inhibition of pro-inflammatory cytokines. On the other hand, both metabolic health and immune health benefit from the practice of physical activity, which reduces the risk of infectious complications. Thus, regular physical exercise appears as a preventive measure in the defense of the host against viral infection [72].

Muscle contraction is responsible for the transient increase in circulating levels of IL-6 cytokine, in proportion to the duration of physical activity and the amount of muscle mass recruited. The elevation of this interleukin seems to be followed by increases in antinflammatory cytokines, such as IL-10, released by cells of innate immunity and responsible for promoting an antinflammatory environment, inhibiting inflammatory mediators to limit tissue damage. This effect may be beneficial in cases of chronic inflammation, such as obesity, T2DM and MS, and may reduce the risk of a pathogenic inflammatory response such as the cytokine storm present in severe COVID-19 [40, 59].

In addition, IL-10 is associated with enhanced insulin sensibility and glycemic metabolism [40]. Physical practice is able to reduce the excessive concentration of pro-inflammatory adipocin leptin and improve sensitivity to leptin and insulin [72, 73]. In patients with T2DM and new coronavirus infection, good glycemic control has been associated with better prognosis in COVID-19 [59].

Thus, physical exercise is shown to be an immunomodulatory and non-pharmacological intervention, achieving positive immunomodulation through exercises of light to moderate intensity [72]. Through exercise, there is an improvement in the response to infection in obese individuals, due to immune and cellular restoration [74]. Although COVID-19 is not primarily a metabolic disease, there is a need to maintain metabolic control of glucose, lipid levels and blood pressure in order to prevent metabolic and cardiovascular complications, as well as to reduce the local inflammatory response and block the virus entering the cells [75].

As seen, innate immunity has an important role in the pathogenesis of COVID-19 and ARDS, due to inflammatory cascades, recruitment of neutrophils, macrophages and DC cells and increased production of ROSs. In turn, by the modulation mechanism of chemokine production, physical training and therapeutic exercises can attenuate alveolar neutrophilia in the face of lung injury [41].

Furthermore, the expression of the extracellular superoxide dismutase enzyme (EcSOD), an important antioxidant in the body and highly present in the lungs, is enhanced by resistance physical activity and was associated with inhibition of endothelial activation and inflammatory adhesion, with potential benefit to reduce oxidative stress and tissue damage in COVID-19 [41]. The amplification of the antioxidant defense generated by routine physical activity also contributes to immunological surveillance [13].

Moreover, Womack, Nagelkirk and Coughlin [76] point out that through the intensity of physical exercise, a change in the potential for coagulation, platelet aggregation and fibrinolysis can be seen. As an example, there is long-term training through aerobic exercises where a decrease in the clotting potential is observed in healthy individuals. Thus, it is suggested that the practice of physical exercises contributes to reducing the risk of ischemic events depending on their intensity and duration [76] and may contribute to attenuating coagulation disorders associated with SARSCoV-2 infection.

The anti-inflammatory, antioxidant and endothelial activation inhibitor benefits may also be linked to the reduction in hypercoagulability related to COVID-19. This is because, as previously mentioned, the exacerbated activation of the immune system increases the expression of the tissue factor and, consequently, the predisposition to the formation of thrombi; in addition, metabolic disorders, oxidant stress and changes in senescence positively stimulate the vasoconstrictor axis ECA/Ang/AT1R, contributing to endothelial imbalances [46, 47, 49-53, 69]. Therefore, the immunometabolic improvements promoted by physical exercise may help in the control of coagulation disorders in the COVID-19 (Fig. 4).

Finally, in view of the quarantine status adopted in several countries as a measure to prevent and control the spread of SARS-CoV-2 during the COVID-19 pandemic, social isolation and restrictions on the movement of people reduced the practice of physical activity, predisposing the population to 
Fig. 4 Benefits of regular moderate-intensity physical activity on factors that influence the response against to COVID19. Source: The Authors (2020)

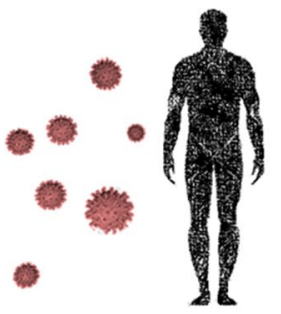

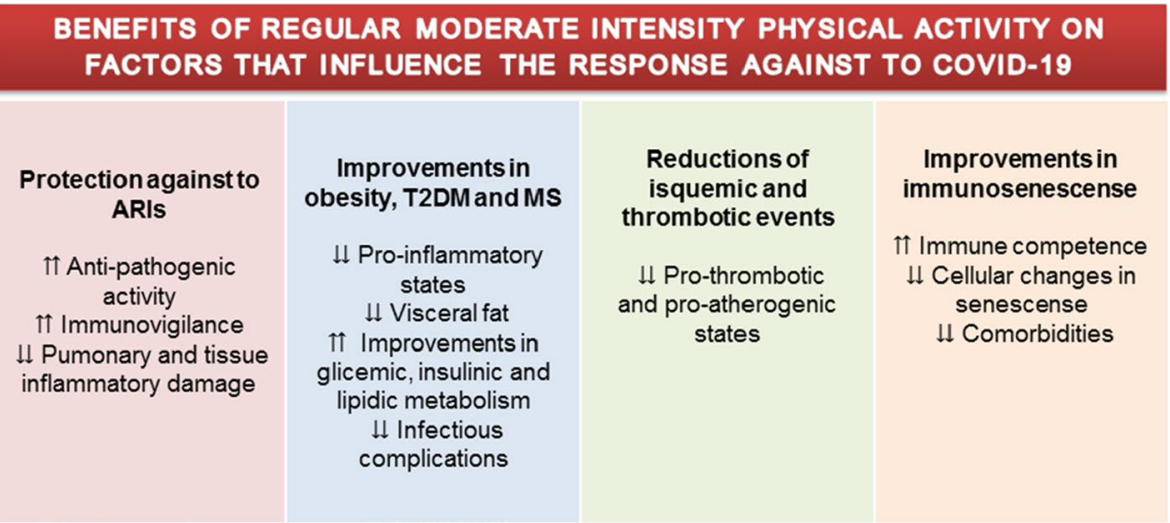

adopt sedentary behavior [77]. Social distancing is a sine qua non in reducing the speed of contagion of COVID-19 and associated deaths. However, due to these measures, sports clubs, gyms and fitness spaces have suspended their activities in order to reduce agglomerations; thus, difficulties regarding physical exercise were imposed [78].

Therefore, despite being one of the main strategies against COVID-19, social isolation has been related to behavioral and physiological changes, including the increased prevalence of sedentarism and eating disorders (food compulsion, hyperphagia), resulting in negative consequences for metabolic health, such as weight gain, growth of fat tissue, hyperglycemia and insulin resistance and loss of muscle tissue [59]. Since this condition can harm the body's defenses and contribute significantly to the reduction in individuals' physical condition, functional and health loss, the adoption of healthy habits and an exercise routine can help in maintaining health $[59,69]$.

It is significant to consider that contexts like this increase the susceptibility to stressful events and elevations of glucocorticoids (cortisol), with consequent inhibition of the functions of NK cells and TCD8 + lymphocytes in the antiviral response. However, good physical conditioning was associated with lower risks of reactivation of latent viral infections in situations of isolation and confinement, indicating a favored immune system compared to individuals with less physical fitness [16].

Physical activity is considered a non-medication practice for the prevention and treatment of diseases of psychological, physical and/or metabolic origin [78]. Regular physical exercise should be encouraged during social isolation as a preventive measure for health, given that exercise is essential during the period of fight against the spread of coronavirus [69].

The American College of Sports Medicine (ACSM) recommends that the practice of moderate physical exercise should be maintained during the quarantine period, since it helps in the immune reinforcement against SARS-CoV-2. The WHO recommends that asymptomatic and healthy individuals should exercise at least $150 \mathrm{~min}$ per week for adults and 300 min per week for children and adolescents. These times can be distributed during the days of the week and according to the person's routine [69]. It is important to emphasize that physical activity should be interrupted and a health professional should be consulted in case symptoms such as fever, dyspnea at rest and dry cough are manifested, because these symptoms can be related to COVID-19 [69, 79].

In social isolation, the home environment has become the ideal and necessary place for physical activity. Activities that are satisfactory and that allow better exploring the home space should be sought. Activities of daily living such as organization of spaces, cleaning and maintenance also help in coping with COVID-19. In environments with children, playing and exercising with them is a great way to promote energy expenditure, thus leaving the beginning of sedentary rest. Meditation, stretching and relaxation are allies in combating a sedentary lifestyle. It is important to avoid long rest periods, which should be intercalated with active practices [69].

The ACSM has published guidelines for moderate-intensity activities that can be practiced during the pandemic period, including aerobic exercises and strength training, indoors, like at home, or outdoors, when permitted by 
government authorities. Options for aerobic activities to be performed at home include walk briskly around the house, up- and downstairs, dancing and jumping rope. When possible, walking or running outdoors, cycling, gardening work and family games are interesting alternatives, as long as infection prevention measures are maintained [79].

Among the strength activities, ACSM indicates downloading a strength workout app that does not require any equipment and suggests exercises such as squat, sit-ups, push-ups, lunges and yoga practice, which can also help in anxious states [79]. Oliveira Neto et al. [80] suggest resistance exercises based on ACSM recommendations to be performed at home, including exercises involving the muscles of the lower body, upper body and limbs, and lower limbs, which can be adapted for beginners in physical practice or experienced people.

Activities that make use of the individual's own body weight, associated with resistance training as well as the use of elastic bands, provide excellent health results, results similar to those achieved by traditional gyms. Thus, objects such as backpacks, books, market bags and water bottles can be used as an auxiliary tool in resistance physical activity. Exercises such as squats, jumping jacks and going up and down steps can be effective in physical training [78].

As for the intensity and volume of physical exercise practices, these must be moderate, as they exceed both the volume and the intensity, effects such as momentary immunosuppression are achieved, thus providing greater vulnerability as to the contagion of the novel coronavirus. If individuals want to practice high-intensity exercises, a reduction in exercise volume should be adopted as a preventive measure, in order to avoid strenuous exercises [78].

In addition, technological tools can contribute to the better performance of these activities in the home environment, as video calls with a physical education professional facilitate the orientation of the exercises to be performed, providing support that has, as a consequence, better results and greater safety in the execution of the exercises. Regardless of whether or not you are in the risk group for COVID-19, regular exercise, according to the ACSM, should be regularly performed, given that it aims to improve the immune system, reduce stress perceived and decrease anxiety disorders [78].

\section{Conclusion}

There are still gaps in the knowledge regarding the pathogenic mechanisms involved in SARS-CoV-2 infection. However, there is consensus in the scientific literature about the important involvement of the immune system in the susceptibility, progression and outcome of COVID-19. The imbalance in innate and adaptive immune responses, characterized mainly by changes such as cytokine storm and lymphopenia, in addition to the disorders in coagulation- and host-related conditions, including obesity, metabolic syndrome and aging (immunosenescence), is among the factors notoriously associated with a worse prognosis of infection.

The benefits of exercise-regular and at appropriate intensity levels-for the immune system in respiratory infections such as COVID-19 include increased immunovigilance and improved immune competence, which help in the control of pathogens, a fact that becomes more important considering the immunosenescence and susceptibility of the elderly population to severe infection. Other favorable effects in relation to host factors, such as prevention or reduction of overweight, increased physical and cardiopulmonary conditioning, attenuation of the systemic pro-inflammatory and pro-thrombotic states, decrease in oxidative stress, improvements in glycemic, insulinic and lipidic metabolisms, besides the enhancement of the vaccination response, also indicate how adequately physical activity can help the organism's immune response against COVID-19.

In the COVID-19 pandemic situation, adopting mitigation practices is an essential strategy to reduce the risks related to the novel coronavirus infection. These interventions include the use of personal protective equipment (PPE), adherence to hygiene procedures and social isolation measures, as well as actions that lead to a healthier lifestyle, minimize stress factors and strengthen the immune system, such as regular physical activity.

However, remaining active at appropriate levels seems to be a challenge in a context of confinement and social isolation, which emphasizes the importance of developing training with recommendations adapted to the new routine of the population. Fortunately, there are viable alternatives for performing physical exercises in restricted environments, enabling the population to enjoy the advantages of physical training for health in the context of OVID-19.

Finally, future studies that deepen the relationship between physical activity and infection by SARS-CoV-2, including the influences of exercise on metabolic and immunological disorders present in COVID-19, will certainly be relevant in view of the probable benefits already mentioned and considering the impacts of infection by the novel coronavirus in the global context. Faced with the possibility of new pandemics by previously unknown microorganisms, without totally effective prevention measures, vaccines or specific treatments of proven efficacy, the host organism's capacity against infections becomes the most important line of defense, thus emphasizing the importance of investing in lifestyle habits that promote health and well-being, such as the practice of physical activity. 
Authors' contributions DTRS had the idea for the article. ES, KKSF e MRB performed the literature search, data analysis and translation. MPS drafted and revised the work.

Funding Not applicable.

\section{Compliance with ethical standards}

Conflict of interest The authors declare that they have no conflict of interest.

Human and animal rights statement and informed consent This article does not contain any studies with human participants or animals performed by any of the authors, and informed consent is not a standard required.

\section{References}

1. Cardoso AM. The persistence of acute respiratory infections as a Public Health problem. Cad Saúde Pública. 2010;26(7):1270-1. https://doi.org/10.1590/S0102-311X2010000700001.

2. Cintra OAL, Arruda E. Respiratory viral infections in immunocompromised patients. Medicina (Ribeirão Preto Online). 1998;32(2):129. https://doi.org/10.11606/issn.2176-7262.v32i2 p129-137.

3. Heikkinen T, Järvinen A. The common cold. Lancet. 2003;361(9351):51-9. https://doi.org/10.1016/S0140 $-6736(03) 12162-9$.

4. Veronesi R, Focaccia R. Tratado de infectologia. 5th ed. São Paulo (SP): Atheneu; 2015.

5. WHO. Scientific Group on Viral Respiratory Diseases \& World Health Organization. Viral respiratory diseases: report of a WHO scientific group [on viral respiratory diseases; Geneva, 2-6 April 1979]. Geneva; 1980.63 p. (Technical report series/World Health Organization).

6. WHO. World Health Organization. Coronavirus disease (COVID19) Pandemic [Internet]. 2020. https://www.who.int/emergencies/ diseases/novel-coronavirus-2019. Accessed 19 July 2020.

7. Liu J, Li S, Liu J, et al. Longitudinal characteristics of lymphocyte responses and cytokine profiles in the peripheral blood of SARSCoV-2 infected patients. EBioMedicine. 2020;55:102763. https:// doi.org/10.1016/j.ebiom.2020.102763.

8. Prompetchara E, Ketloy C, Palaga T. Immune responses in COVID-19 and potential vaccines: lessons learned from SARS and MERS epidemic. Asian Pac J Allergy Immunol. 2020;38(1):1-9. https://doi.org/10.12932/AP-200220-0772.

9. Li X, Geng M, Peng Y, Meng L, Lu S. Molecular immune pathogenesis and diagnosis of COVID-19. J Pharm Anal. 2020;10(2):102-8. https://doi.org/10.1016/j.jpha.2020.03.001.

10. Simpson RJ, Katsanis E. The immunological case for staying active during the COVID-19 pandemic. Brain Behav Immun. 2020. https://doi.org/10.1016/j.bbi.2020.04.041.

11. Elenkov IJ, Chrousos GP, Wilder RL. Neuroendocrine regulation of IL-12 and TNF-alpha/IL-10 balance. Clinical implications. Ann NY Acad Sci. 2000;917:94-105. https://doi. org/10.1111/j.1749-6632.2000.tb05374.x.

12. Nieman DC, Wentz LM. The compelling link between physical activity and the body's defense system. J Sport Health Sci. 2019;8(3):201-17. https://doi.org/10.1016/j.jshs.2018.09.009.

13. Laddu DR, Lavie CJ, Phillips SA, Arena R. Physical activity for immunity protection: inoculating populations with healthy living medicine in preparation for the next pandemic. Prog Cardiovasc Dis. 2020. https://doi.org/10.1016/j.pcad.2020.04.006.

14. Pedersen BK, Hoffman-Goetz L. Exercise and the immune system: regulation integration and adaption. Physiol Rev. 2000;80(3):1055-81. https://doi.org/10.1152/physr ev.2000.80.3.1055.

15. Leandro CG, Castro RM, Nascimento E, Pithon-Curi TC, Curi R. Adaptative mechanisms of the immune system in response to physical training. Rev Bras Med Esporte. 2007;13(5):343-8. https ://doi.org/10.1590/S1517-86922007000500012.

16. Simpson RJ, Katsanis E. The immunological case for staying active during the COVID-19 pandemic. Brain Behav Immunity. 2020. https://doi.org/10.1016/j.bbi.2020.04.041.

17. Walsh NP, Gleeson M, Pyne DB, et al. Position statement part two: maintaining immune health. Exerc Immunol Rev. 2011;17:64-103.

18. Elenkov IJ, Chrousos GP, Wilder RL. Neuroendocrine regulation of IL-12 and TNF-alpha/IL-10 balance. Clinical implications. Ann NY Acad Sci. 2006;917(1):94-105. https://doi. org/10.1111/j.1749-6632.2000.tb05374.x.

19. Cannon JG. Inflammatory cytokines in nonpathological states. News Physiol Sci. 2000;15(6):298-303. https://doi.org/10.1152/ physiologyonline.2000.15.6.298.

20. Terra R, da Silva SAG, Pinto VS, Dutra PML. Efeito do exercício no sistema imune: resposta, adaptação e sinalização celular. Rev Bras Med Esporte. 2012;18(3):208-14. https://doi.org/10.1590/ S1517-86922012000300015.

21. Brickson S, Hollander J, Corr DT, Ji LL, Best TM. Oxidant production and immune response after stretch injury in skeletal muscle. Med Sci Sports Exerc. 2001;33(12):2010-5. https://doi. org/10.1097/00005768-200112000-00006.

22. Lavie CJ, Lee D, Sui X, et al. Effects of running on chronic diseases and cardiovascular and all-cause mortality. Mayo Clin Proc. 2015;90(11):1541-52. https://doi.org/10.1016/j.mayoc p.2015.08.001.

23. Wolach B, Gavrieli R, Ben-Dror SG, Zigel L, Eliakim A, Falk B. Transient decrease of neutrophil chemotaxis following aerobic exercise. Med Sci Sports Exerc. 2005;37(6):949-54.

24. Matthews CE, Ockene IS, Freedson PS, Rosal MC, Merriam PA, Hebert JR. Moderate to vigorous physical activity and risk of upper-respiratory tract infection. Med Sci Sports Exerc. 2002;34(8):1242-8. https://doi.org/10.1097/00005768-20020 8000-00003.

25. Zhou G, Liu H, He M, et al. Smoking, leisure-time exercise and frequency of self-reported common cold among the general population in northeastern China: a cross-sectional study. BMC Public Health. 2018;18(1):294. https://doi.org/10.1186/s 1288 9-018-5203-5.

26. Gleeson M, McFarlin B, Flynn M. Exercise and Toll-like receptors. Exerc Immunol Rev. 2006;12:34-53.

27. Dela F, Mikines KJ, Von Linstow M, Galbo H. Heart rate and plasma catecholamines during $24 \mathrm{~h}$ of everyday life in trained and untrained men. J Appl Physiol. 1992;73(6):2389-95. https://doi. org/10.1152/jappl.1992.73.6.2389.

28. Malm C, Sjödin B, Sjöberg B, et al. Leukocytes, cytokines, growth factors and hormones in human skeletal muscle and blood after uphill or downhill running: leukocytes, cytokines and hormones in response to physical exercise. J Physiol. 2004;556(3):983-1000. https://doi.org/10.1113/jphysiol.2003.056598.

29. Oshida Y, Yamanouchi K, Hayamizu S, Sato Y. Effect of acute physical exercise on lymphocyte subpopulations in trained and untrained subjects. Int J Sports Med. 1988;09(02):137-40. https ://doi.org/10.1055/s-2007-1024995.

30. Hansen JB, Wilsgard L, Osterud B. Biphasic changes in leukocytes induced by strenuous exercise. Europ J Appl Physiol. 1991;62(3):157-61. https://doi.org/10.1007/BF00643735. 
31. Timmons BW, Cieslak T. Human natural killer cell subsets and acute exercise: a brief review. Exerc Immunol Rev. 2008;14:8-23.

32. Navalta J, Sedlock D, Park K-S. Effect of exercise intensity on exercise-induced lymphocyte apoptosis. Int J Sports Med. 2007;28(6):539-42. https://doi.org/10.1055/s-2006-955898.

33. Lu R, Zhao X, Li J, et al. Genomic characterisation and epidemiology of 2019 novel coronavirus: implications for virus origins and receptor binding. Lancet. 2020;395(10224):565-74. https://doi. org/10.1016/S0140-6736(20)30251-8.

34. Wang P-H, Cheng Y. Increasing host cellular receptor-angiotensin-converting enzyme 2 (Ace2) expression by coronavirus may facilitate 2019-ncov infection. bioRxiv. 2020. https://doi. org/10.1101/2020.02.24.963348.

35. Huang C, Wang Y, Li X, et al. Clinical features of patients infected with 2019 novel coronavirus in Wuhan, China. Lancet. 2020;395(10223):497-506. https://doi.org/10.1016/S0140 -6736(20)30183-5.

36. Mello C, Aguayo E, Rodriguez M, et al. Multiple classes of antiviral agents exhibit in vitro activity against human rhinovirus type c. Antimicrobial Agents Chemother. 2014;58(3):1546-55. https:// doi.org/10.1128/AAC.01746-13.

37. Cao X. COVID-19: immunopathology and its implications for therapy. Nat Rev Immunol. 2020;20(5):269-70. https://doi. org/10.1038/s41577-020-0308-3.

38. Li H, Liu L, Zhang D, et al. SARS-CoV-2 and viral sepsis: observations and hypotheses. Lancet. 2020;395(10235):1517-20. https ://doi.org/10.1016/S0140-6736(20)30920-X.

39. Li G, Fan Y, Lai Y, et al. Coronavirus infections and immune responses. J Med Virol. 2020;92(4):424-32. https://doi. org/10.1002/jmv.25685.

40. Gleeson M, Bishop NC, Stensel DJ, Lindley MR, Mastana SS, Nimmo MA. The anti-inflammatory effects of exercise: mechanisms and implications for the prevention and treatment of disease. Nat Rev Immunol. 2011;11(9):607-15. https://doi. org/10.1038/nri3041.

41. Yan Z, Spaulding HR. Extracellular superoxide dismutase, a molecular transducer of health benefits of exercise. Redox Biol. 2020;32:101508. https://doi.org/10.1016/j.redox.2020.101508.

42. Dolin R. Infecções respiratórias virais comuns. In: Kasper D, Fauci A, Hauser S, et al., editors. Medicina interna de Harrison. 19th ed. Porto Alegre: AMGH; 2017. p. 5010-26.

43. Sanchis-Gomar F, Lavie CJ, Mehra MR, Henry BM, Lippi G. Obesity and outcomes in covid-19: when an epidemic and pandemic collide. Mayo Clin Proc. 2020;95(7):1445-53. https://doi. org/10.1016/j.mayocp.2020.05.006.

44. Tang N, Li D, Wang X, Sun Z. Abnormal coagulation parameters are associated with poor prognosis in patients with novel coronavirus pneumonia. J Thromb Haemost. 2020;18(4):844-7. https:// doi.org/10.1111/jth.14768.

45. Han H, Yang L, Liu R, et al. Prominent changes in blood coagulation of patients with SARS-CoV-2 infection. Clin Chem Lab Med. 2020. https://doi.org/10.1515/cclm-2020-0188.

46. Spiezia L, Boscolo A, Poletto F, et al. Covid-19-related severe hypercoagulability in patients admitted to intensive care unit for acute respiratory failure. Thromb Haemost. 2020. https://doi. org/10.1055/s-0040-1710018.

47. Henry BM, Vikse J, Benoit S, Favaloro EJ, Lippi G. Hyperinflammation and derangement of renin-angiotensin-aldosterone system in COVID-19: a novel hypothesis for clinically suspected hypercoagulopathy and microvascular immunothrombosis. Clin Chim Acta. 2020;507:167-73. https://doi.org/10.1016/j. cca.2020.04.027.

48. Minasyan H, Flachsbart F. Blood coagulation: a powerful bactericidal mechanism of human innate immunity. Int Rev Immunol. 2019;38(1):3-17. https://doi.org/10.1080/08830185.2018.15330 09.
49. Vivas D, Roldán V, Esteve-Pastor MA, et al. Recommendations on antithrombotic treatment during the covid-19 pandemic Position statement of the Working Group on Cardiovascular Thrombosis of the Spanish Society of Cardiology. Rev Esp Cardiol. 2020. https ://doi.org/10.1016/j.recesp.2020.04.006.

50. Hamming I, Timens W, Bulthuis MLC, Lely AT, Navis GJ, van Goor H. Tissue distribution of ACE2 protein, the functional receptor for SARS coronavirus A first step in understanding SARS pathogenesis. J Pathol. 2004;203(2):631-7. https://doi. org/10.1002/path.1570.

51. Gonsalez SR, Ferrão FM, de Souza AM, Lowe J, Morcillo LSL. Inappropriate activity of local renin-angiotensin-aldosterone system during high salt intake: impact on the cardiorenal axis. J Bras Nefrol. 2018;40(2):170-8. https://doi. org/10.1590/2175-8239-jbn-3661.

52. Dalan R, Bornstein SR, El-Armouche A, et al. The ACE-2 in COVID-19: foe or Friend? Horm Metab Res. 2020;52(05):25763. https://doi.org/10.1055/a-1155-0501.

53. Tikellis C, Thomas MC. Angiotensin-Converting Enzyme 2 (ACE2) is a key modulator of the renin angiotensin system in health and disease. Int J Peptides. 2012;2012:1-8. https://doi. org/10.1155/2012/256294.

54. Li M-Y, Li L, Zhang Y, Wang X-S. Expression of the SARSCoV-2 cell receptor gene ACE2 in a wide variety of human tissues. Infect Dis Poverty. 2020;9(1):45. https://doi.org/10.1186/ s40249-020-00662-x.

55. Sargiacomo C, Sotgia F, Lisanti MP. COVID-19 and chronological aging: senolytics and other anti-aging drugs for the treatment or prevention of corona virus infection? Aging (Albany NY). 2020;12(8):6511-7. https://doi.org/10.18632/aging.103001.

56. Guidi L, Tricerri A, Frasca D, Vangeli M, Errani AR, Bartoloni C. Psychoneuroimmunology and aging. Gerontology. 1998;44(5):247-61. https://doi.org/10.1159/000022021.

57. Shaw AC, Joshi S, Greenwood H, Panda A, Lord JM. Aging of the innate immune system. Curr Opin Immunol. 2010;22(4):507-13. https://doi.org/10.1016/j.coi.2010.05.003.

58. Ewers I, Rizzo LV, Kalil F. Imunologia e envelhecimento. Einstein. 2008;6(Suppl. 01):S13-20.

59. Ayres JS. A metabolic handbook for the COVID-19 pandemic. Nat Metab. 2020. https://doi.org/10.1038/s42255-020-0237-2.

60. Ryan PM, Caplice NM. Is adipose tissue a reservoir for viral spread, immune activation and cytokine amplification in COVID19. Obesity. 2020. https://doi.org/10.1002/oby.22843.

61. Andersen CJ, Murphy KE, Fernandez ML. Impact of obesity and metabolic syndrome on immunity. Adv Nutr. 2016;7(1):66-75. https://doi.org/10.3945/an.115.010207.

62. Milner JJ, Beck MA. The impact of obesity on the immune response to infection. Proc Nutr Soc. 2012;71(2):298-306. https ://doi.org/10.1017/s0029665112000158.

63. Francisqueti FV, Nascimento AF, Correa CR. Obesity, inflammation and metabolic complications. Nutrire. 2015;40(1):81-9. https ://doi.org/10.4322/2316-7874.016213.

64. Prado WL, Lofrano MC, Oyama LM, Dâmaso AR. Obesity and inflammatory adipokines: practical implications for exercise prescription. Rev Bras Med Esporte. 2009;15(5):378-83. https://doi. org/10.1590/s1517-86922009000600012.

65. Trim W, Turner J, Thompson D. Parallels in immunometabolic adipose tissue dysfunction with ageing and obesity. Front Immunol. 2018;9:169. https://doi.org/10.3389/fimmu.2018.00169.

66. Petrakis D, Margină D, Tsarouhas K, et al. Obesity-a risk factor for increased COVID-19 prevalence, severity and lethality (Review). Mol Med Rep. 2020. https://doi.org/10.3892/ mmr.2020.11127.

67. Mraz M, Haluzik M. The role of adipose tissue immune cells in obesity and low-grade inflammation. J Endocrinol. 2014;222(3):R113-27. https://doi.org/10.1530/JOE-14-0283. 
68. Nieman DC. Coronavirus Disease-2019: a tocsin to our aging, unfit, corpulent, and immunodeficient society. J Sport Health Sci. 2020. https://doi.org/10.1016/j.jshs.2020.05.001.

69. Ferreira MJ, Irigoyen MC, Consolim-Colombo F, Angelis K. Physically active lifestyle as an approach to confronting COVID19. Arq Bras Cardiol. 2020. https://doi.org/10.36660/abc. 20200 235.

70. Damiot A, Pinto AJ, Turner JE, Gualano B. Immunological implications of physical inactivity among older adults during the covid19 pandemic. Gerontology. 2020. https://doi.org/10.1159/00050 9216.

71. Ferrer M, Capó X, Martorell M, Busquets-Cortés C, Bouzas C, Carreres $\mathrm{S}$, et al. Regular practice of moderate physical activity by older adults ameliorates their anti-inflammatory status. Nutrients. 2018;10(11):1780. https://doi.org/10.3390/nu10111780.

72. Luzi L, Radaelli MG. Influenza and obesity: its odd relationship and the lessons for COVID-19 pandemic. Acta Diabetol. 2020;57(6):759-64. https://doi.org/10.1007/s00592-020-01522-8.

73. Monteiro EC, Navarro F. Leptin, obesity and physical exercise. RBONE-Brazilian Journal of Obesity, Nutrition and Weight Loss. 2010. http://www.rbone.com.br/index.php/rbone/article/view/206. Accessed 18 May 2020.

74. Honce R, Schultz-Cherry S. Impact of obesity on influenza a virus pathogenesis, immune response, and evolution. Front Immunol. 2019;10:1071. https://doi.org/10.3389/fimmu.2019.01071.

75. Bornstein SR, Dalan R, Hopkins D, Mingrone G, Boehm BO. Endocrine and metabolic link to coronavirus infection. Nat Rev Endocrinol. 2020;16(6):297-8. https://doi.org/10.1038/s4157 4-020-0353-9.
76. Womack CJ, Nagelkirk PR, Coughlin AM. Exercise-induced changes in coagulation and fibrinolysis in healthy populations and patients with cardiovascular disease. Sports Med. 2003;33(11):795-807. https://doi.org/10.2165/00007256-20033 3110-00002.

77. Fallon K. Exercise in the time of COVID-19. Aust J Gen Pract. 2020. https://doi.org/10.31128/AJGP-COVID-13.

78. Raiol RA. Praticar exercícios físicos é fundamental para a saúde física e mental durante a Pandemia da COVID-19. Braz J Health Rev. 2020. https://doi.org/10.34119/bjhrv3n2-124.

79. ACSM. American College of Sports Medicine. Staying Active During the Coronavirus Pandemic [Internet]. https://www. exerciseismedicine.org/assets/page_documents/EIM_Rx\%20 for\%20Health_\%20Staying\%20Active\%20During\%20Coronavi rus\%20Pandemic.pdf. Accessed 10 July 2020.

80. De Oliveira Neto L, Elsangedy HM, Tavares VDDO, Teixeira CVLS, Behm DG, Da Silva-Grigoletto ME. TrainingInHomeHome-based training during COVID-19 (Sars-cov2) pandemic: physical exercise and behavior-based approach. RBFE. 2020;19(2):9. https://doi.org/10.33233/rbfe.v19i2.4006.

Publisher's Note Springer Nature remains neutral with regard to jurisdictional claims in published maps and institutional affiliations. 